\title{
Size of the abductor hallucis muscle in older women with hallux valgus
}

\author{
Karen J Mickle ${ }^{1 *}$, Christopher J Nester ${ }^{2}$ \\ From 4th Congress of the International Foot and Ankle Biomechanics (i-FAB) Community \\ Busan, Korea. 8-11 April 2014
}

Toe deformities are highly prevalent in older people with up to $74 \%$ of older men and women having some degree of hallux valgus [1]. Despite the well documented hypotheses that atrophied, or weak toe flexor muscles are associated with the formation of toe deformities [2], there has been little evidence to support this theory. Only one study has directly compared the toe flexor strength of individuals with toe deformities to those without, revealing that older people with hallux valgus have reduced hallux strength compared to those without the deformity [3]. Therefore, to further investigate the pathomechanics of hallux valgus, this study aimed to determine whether the size of the abductor hallucis muscle differed in older women with and without hallux valgus deformity.

Forty-four older adults (60+ years) were recruited to participate in the study. Each participant had their feet assessed by the Chief Investigator (KJM), with hallux valgus severity rated using the Manchester Scale [4]. The abductor hallucis muscle was imaged using a GE Venue 40 US with a 6-9 MHz transducer [5]. Muscle cross-sectional area (CSA) was measured using Image J software with the assessor blinded to group allocation. Ten participants (all women) were classified as having moderate or severe hallux valgus and their muscle size was compared to 10 age and BMI matched women without any hallux deformity.

The older women with moderate-severe hallux valgus were found to have a significantly reduced cross-sectional area of the abductor hallucis muscle ( $<0.05$; Figure 1$)$. This may suggest that muscle weakness and atrophy is associated with the development or progression of hallux valgus, however further longitudinal studies are required to confirm this notion. Further research is also required to determine whether strengthening the toe flexor muscles

\footnotetext{
* Correspondence: kmickle@uow.edu.au

'Biomechanics Research Laboratory, University of Wollongong, NSW, 2522, Australia

Full list of author information is available at the end of the article
}

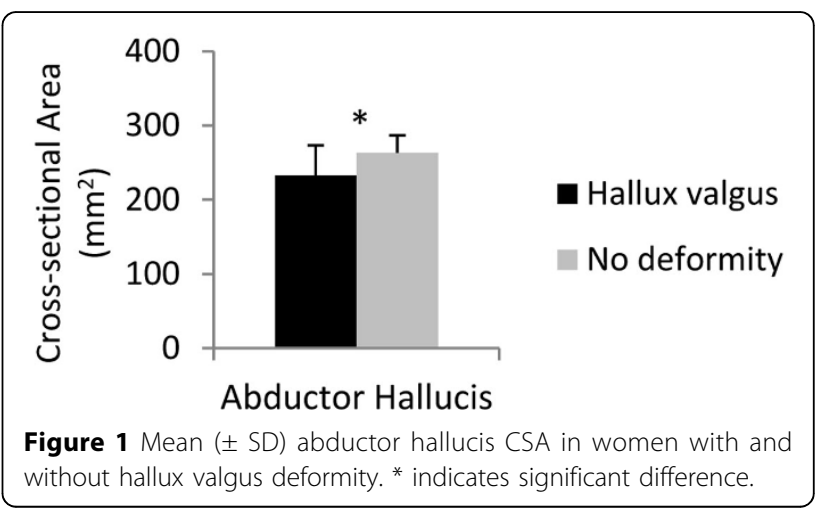

results in hypertrophic changes to muscle morphology and these results highlight the need to investigate whether strengthening the intrinsic toe muscles could reduce the incidence and severity of toe deformities.

\section{Authors' details}

'Biomechanics Research Laboratory, University of Wollongong, NSW, 2522, Australia. ${ }^{2}$ Centre for Health Sciences Research, University of Salford, Salford, M6 6PU, UK.

\section{Published: 8 April 2014}

\section{References}

1. Dunn JE, et al: Prevalence of foot and ankle conditions in a multiethnic community sample of older adults. Am. J. Epidemiol 2004, 159(5):491-8.

2. Myerson MS, Shereff MJ: The pathological anatomy of claw and hammer toes. J. Bone Joint Surg. Am 1989, 71-A(1):45-9.

3. Mickle KJ, et al: ISB Clinical Biomechanics Award 2009: Toe weakness and deformity increase the risk of falls in older people. Clin. Biomech 2009, 24:787-791.

4. Garrow AP, et al: The grading of hallux valgus. The Manchester Scale. J. Am. Podiatr. Med. Assoc 2001, 91(2):74-8.

5. Mickle $K$, et al: Reliability of ultrasound to measure morphology of the toe flexor muscles. J Foot Ankle Res 2013, 6(1):12.

doi:10.1186/1757-1146-7-S1-A57

Cite this article as: Mickle and Nester: Size of the abductor hallucis muscle in older women with hallux valgus. Journal of Foot and Ankle Research 2014 7(Suppl 1):A57. 